\title{
$d$-WISE GENERATION OF SOME INFINITE GROUPS
}

\author{
ANDREA LUCCHINI, ATTILA MARÓTI, DARREN SEMMEN
}

\begin{abstract}
What is the largest possible size of a subset of $S L(n, \mathbb{Z})$ from which every pair of elements will be a generating set? We prove a general result on generation probabilities in profinite groups that suggests the cardinality of a maximal such subset equals that of the analogous subset of $S L(n, \mathbb{Z} / 2 \mathbb{Z})$.
\end{abstract}

Let $d$ be a positive integer greater than or equal to 2 , and let $G$ be a discrete or profinite group that can be topologically generated by $d$ elements. If there is a largest integer $m$ with the property that there exists an $m$-tuple of elements of $G$ such that any $d$ entries together (topologically) generate $G$ then denote this number by $\mu_{d}(G)$, and otherwise set $\mu_{d}(G)$ equal to $\infty$. If $G$ cannot be generated by $d$ elements then set $\mu_{d}(G)=0$.

A motivation for studying $\mu_{d}(G)$ is given by Theorem 12 .

Another reason why the function $\mu_{d}(G)$ may be interesting is that it can be computed explicitly for certain groups $G$. For if $G$ is any of the groups $S_{n}$ for sufficiently large odd $n$, or $A_{n}$ for sufficiently large $n$ congruent to 2 modulo 4 , or $G L(n, q), P G L(n, q), S L(n, q), P S L(n, q)$ for $n$ at least 12 and not congruent to 2 modulo 4 , or $M_{11}$, or $M_{23}$, then there is an explicit and exact formula for $\mu_{d}(G)$. (For $d=2$ this formula is found in [2], [3] and [4] respectively where it is also shown that $\mu_{2}(G)=\sigma(G)$ where $\sigma(G)$ is defined in the first paragraph of Section 2. Now apply Lemma 2 to conclude that $\mu_{d}(G)=(d-1) \mu_{2}(G)$.)

If $n$ is a positive integer greater than or equal to 2 then the group $S L(n, \mathbb{Z})$ is 2 -generated. Hence, it makes sense to investigate $\mu_{d}(S L(n, \mathbb{Z}))$. Since $S L(n, \mathbb{Z} / 2 \mathbb{Z})$ is a factor group of $S L(n, \mathbb{Z})$, we certainly have $\mu_{d}(S L(n, \mathbb{Z})) \leq \mu_{d}(S L(n, \mathbb{Z} / 2 \mathbb{Z}))$. This, Lemma 2, Fact 8 taken from [3], and a bit of computation yields that $\nu_{d}(G)$ defined by

$$
\left(b \cdot \mu_{d}(G)\right) /\left((d-1)\left(\prod_{\substack{i=1 \\ b \nmid i}}^{n-1}\left(2^{n}-2^{i}\right)+\lfloor N(b) / 2\rfloor\right)\right)
$$

is less than $1+2^{-n+1}$ for $G=S L(n, \mathbb{Z})$ and $n \geq 12$ where $b$ is the smallest prime divisor of $n$, the integer $N(b)$ is the number of subspaces of a fixed $n$-dimensional vector space over the field of 2 elements and $\lfloor x\rfloor$ denotes the largest integer less than or equal to $x$. Moreover, by Fact 8 taken from [3], if the answer to the following question is affirmative for $n \geq 12$, then we also have $\nu_{d}(S L(n, \mathbb{Z})) \geq 1$ for $n \geq 12$.

Question 1. Is it true that $\mu_{d}(S L(n, \mathbb{Z}))=\mu_{d}(S L(n, \mathbb{Z} / 2 \mathbb{Z}))$ for all integers $n$ and $d$ greater than or equal to 2 ?

Everything we do in this paper is intended to suggest that the answer should be "yes" rather than "no". We prove that for $n \geq 12$ the answer is "yes" if we replace $S L(n, \mathbb{Z})$ by its profinite completion, and so $1 \leq \nu_{d}(S \widehat{L(n, \mathbb{Z})})<1+2^{-n+1}$

\footnotetext{
${ }^{0}$ Research of the second author was partially supported by NSF Grant DMS 0140578 and by OTKA T049841.

Date: 1st of May 2008.
} 
for $n \geq 12$ (with equality on the left-hand-side if (but not necessarily only if) $n$ is not congruent to 2 modulo 4). Furthermore, when $n \geq 3$, the probability is positive that a random $\mu_{d}(\widehat{S(n, \mathbb{Z})})$-tuple will have the property that any $d$ entries will

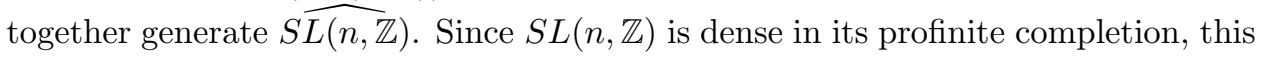
suggests that the answer to our question is "yes", though it hardly proves it.

\section{Computing $\mu_{d}(\widehat{S(n, \mathbb{Z})})$}

For a group $G$ let $\sigma(G)$ denote the minimal cardinality of a covering of $G$, i.e., a collection of proper subgroups whose union is $G$. If $G$ cannot be expressed as a union of proper subgroups, i.e., $G$ is cyclic, then set $\sigma(G)=\infty$.

Our first observation is what allows us to compute explicit formulae for $\mu_{d}$.

Lemma 2. If the non-cyclic group $G$ can be generated by 2 elements, then

$$
(d-1) \mu_{2}(G) \leq \mu_{d}(G) \leq(d-1) \sigma(G) .
$$

Proof. The result is trivial if $\mu_{2}(G)=\infty$. So suppose that $\mu_{2}(G)$ is finite. Suppose $g_{1}, \ldots, g_{n}$ pairwise generate $G$. Let $x$ be a $(d n-n)$-tuple whose first $(d-1)$ entries equal $g_{1}$, whose second $(d-1)$ entries equal $g_{2}$, etc. Then, any $d$ entries of $x$ will generate $G$. The second inequality follows from the fact that, for any $d$ entries of a tuple $\tau$ to generate $G$, if $\mathcal{C}$ is a covering of $G$ then at most $d-1$ entries of $\tau$ can belong to any one element of $\mathcal{C}$.

The simplest case of the discrete general linear group is the only one we can handle.

Lemma 3. $\mu_{d}(S L(2, \mathbb{Z}))=4(d-1)=\mu_{d}(S L(2, \mathbb{Z} / 2 \mathbb{Z}))$.

Proof. Because $S L(2, \mathbb{Z})$ is pairwise generated by the four matrices,

$$
\left(\begin{array}{ll}
1 & 1 \\
0 & 1
\end{array}\right),\left(\begin{array}{ll}
1 & 0 \\
1 & 1
\end{array}\right),\left(\begin{array}{cc}
0 & 1 \\
-1 & 0
\end{array}\right), \text { and }\left(\begin{array}{cc}
0 & 1 \\
-1 & -1
\end{array}\right)
$$

we have $\mu_{2}(S L(2, \mathbb{Z} / 2 \mathbb{Z})) \geq \mu_{2}(S L(2, \mathbb{Z})) \geq 4$. On the other hand, the group $S L(2, \mathbb{Z} / 2 \mathbb{Z})$ is isomorphic to the symmetric group on three letters and so has a minimal covering consisting of the Sylow 3-subgroup and the three Sylow 2-subgroups. Now apply Lemma 2.

For $n \geq 3$ we will move to the profinite completion $S \widehat{L(n, \mathbb{Z})}$ of $S L(n, \mathbb{Z})$. Three of the easy observations can be stated for any profinite group.

Lemma 4. For any profinite group $G$ that can be generated topologically by $d$ elements,

$$
\mu_{d}(G)=\min \left\{\mu_{d}(G / N) \mid N \text { is an open normal subgroup of } G\right\}
$$

Proof. Clearly, $\mu_{d}(G) \leq \mu_{d}(G / N)$ for each open normal subgroup $N$. Suppose that the positive integer $\ell$ is such that $\mu_{d}(G / N) \geq \ell$ for each open normal subgroup $N$. Let $X_{N}$ be the subset of $(G / N)^{\ell}$ whose elements are exactly those tuples from which any choice of $d$ entries will form a set that generates $G / N$. Let $Y_{N}$ be the preimage of $X_{N}$ in $G^{\ell}$. Then each $Y_{N}$ is closed and the intersection of any finite number of the $Y_{N}$ is nonempty. Since $G$ is compact, the intersection is non-empty and so $\mu_{d}(G) \geq \ell$. 
Fact 5 (Neumann, [8]). If $G$ is a group that is the union of finitely many proper subgroups then

$$
\sigma(G)=\min \{\sigma(G / N) \mid N \text { is a finite-index normal subgroup of } G\} .
$$

Lemma 6. For any group $G$ we have both $\mu_{d}(G)=\mu_{d}(G / \Phi(G)$ ) and $\sigma(G)=$ $\sigma(G / \Phi(G))$, where $\Phi(G)$ denotes the Frattini subgroup of $G$.

Note that $S L(n, \mathbb{Z})$ has the congruence subgroup property for $n \geq 3$ (cf. [1] or $[7])$. This is why we next consider groups of the form $S L(n, \mathbb{Z} / N \mathbb{Z})$, where $N$ is a positive integer.

Let $N$ be a positive integer with prime power decomposition $N=p_{1}^{r_{1}} \cdot \ldots \cdot p_{t}^{r_{t}}$. Then, by the Chinese Remainder Theorem, $S L(n, \mathbb{Z} / N \mathbb{Z})=\prod_{i=1}^{t} S L\left(n, \mathbb{Z} / p_{i}^{r_{i}} \mathbb{Z}\right)$. We also have $\Phi(S L(n, \mathbb{Z} / N \mathbb{Z}))=\prod_{i=1}^{t} \Phi\left(S L\left(n, \mathbb{Z} / p_{i}^{r_{i}} \mathbb{Z}\right)\right)$.

Lemma 7. Let $n$ and $N$ be positive integers with $n \geq 5$. Let $\alpha$ denote $\mu_{d}$ or $\sigma$. Then, $\alpha(S L(n, \mathbb{Z} / N \mathbb{Z}))=\min _{1 \leq i \leq t}\left\{\alpha\left(P S L\left(n, \mathbb{Z} / p_{i} \mathbb{Z}\right)\right)\right\}$, where $p_{1}, \ldots, p_{t}$ are the distinct prime divisors of $N$.

Proof. We have

$$
\begin{aligned}
\alpha(S L(n, \mathbb{Z} / N \mathbb{Z})) & =\alpha(S L(n, \mathbb{Z} / N \mathbb{Z}) / \Phi(S L(n, \mathbb{Z} / N \mathbb{Z}))) \\
& =\alpha\left(\prod_{i=1}^{t} S L\left(n, \mathbb{Z} / p_{i}^{r_{i}} \mathbb{Z}\right) / \Phi\left(S L\left(n, \mathbb{Z} / p_{i}^{r_{i}} \mathbb{Z}\right)\right)\right) \\
& =\alpha\left(\prod_{i=1}^{t} \operatorname{PSL}\left(n, \mathbb{Z} / p_{i} \mathbb{Z}\right)\right) \\
& =\min _{1 \leq i \leq t} \alpha\left(\operatorname{PSL}\left(n, \mathbb{Z} / p_{i} \mathbb{Z}\right)\right),
\end{aligned}
$$

where the first equality follows from Lemma 6 , the third equality follows from a result of Weigel [9, Theorem B], and the last equality follows from the fact that the direct summands are non-isomorphic simple groups.

Fact 8 (Theorems 1.1 and 1.2 of [3]). Let $n$ be a positive integer greater than or equal to 12 , let $b$ be the smallest prime divisor of $n$, and let $N(b)$ denote the number of subspaces of the $n$-dimensional vector space over $\mathbb{Z} / p \mathbb{Z}$ which have dimension not divisible by $b$. Then,

$$
\mu_{2}(S L(n, \mathbb{Z} / p \mathbb{Z}))=\frac{1}{b} \prod_{\substack{i=1 \\ b \nmid i}}^{n-1}\left(p^{n}-p^{i}\right)+\lfloor N(b) / 2\rfloor,
$$

where $\lfloor x\rfloor$ denotes the largest integer less than or equal to $x$. Also, $\sigma(S L(n, \mathbb{Z} / p \mathbb{Z}))$ equals $\mu_{2}(S L(n, \mathbb{Z} / p \mathbb{Z}))$ unless $n$ is congruent to 2 modulo 4 and $p$ equals 2 , in which case

$$
\sigma(S L(n, \mathbb{Z} / 2 \mathbb{Z}))=\frac{1}{2} \prod_{\substack{i=1 \\
2 \nmid i}}^{n-1}\left(2^{n}-2^{i}\right)+\lfloor N(2) / 2\rfloor+\frac{2^{n / 2}}{2^{n / 2}+1}\left[\begin{array}{c}
n \\
n / 2
\end{array}\right]_{2},
$$

where $\left[\begin{array}{c}n \\ n / 2\end{array}\right]_{2}$ denotes the number of $(n / 2)$-dimensional subspaces of an $n$-dimensional vector space over $\mathbb{Z} / 2 \mathbb{Z}$.

Theorem 9. Let $n$ be a positive integer greater than or equal to 12 . Then, the following three statements are true.

(1) $\mu_{d}(S \widehat{L(n, \mathbb{Z})})=\mu_{d}(S L(n, \mathbb{Z} / 2 \mathbb{Z}))$.

(2) $\sigma(S L(n, \mathbb{Z}))=\sigma(S \widehat{L(n, \mathbb{Z}))}=\sigma(S L(n, \mathbb{Z} / 2 \mathbb{Z}))$.

(3) If $n$ is not congruent to 2 modulo 4 then

$$
\mu_{d}(S \widehat{L(n, \mathbb{Z})})=(d-1) \mu_{2}(S L(n, \mathbb{Z} / 2 \mathbb{Z})) .
$$


Proof. Remember that $S L(n, \mathbb{Z})$ has the congruence subgroup property when $n \geq 3$.

Fact 5 and Lemma 7 show that $\sigma(S L(n, \mathbb{Z}))$ and $\sigma(S \widehat{L(n, \mathbb{Z}))}$ both equal the minimum of $\sigma(P S L(n, \mathbb{Z} / p \mathbb{Z}))$, where $p$ ranges over all prime natural numbers. By Fact 8 , this minimum occurs when $p=2$.

By Lemmas 4 and $7, \mu_{d}(S \widehat{L(n, \mathbb{Z})})$ will equal the minimum of $\mu_{d}(P S L(n, \mathbb{Z} / p \mathbb{Z}))$, where $p$ ranges over all prime natural numbers. By Lemma 2 and Fact 8, this minimum occurs when $p=2$.

When $n$ is not congruent to 2 modulo 4 , Fact 8 states that $\sigma(S L(n, \mathbb{Z} / 2 \mathbb{Z}))$ equals $\mu_{2}(S L(n, \mathbb{Z} / 2 \mathbb{Z}))$ and the rest of the third statement then follows from Lemma 2.

\section{Generation probabilities in Profinite Groups}

Next we will show that, whenever $n \geq 3$ and $d \geq 2$, the probability is positive that a randomly chosen $\mu_{d}(S \widehat{L(n, \mathbb{Z})})$-tuple with entries from $S \widehat{L(n, \mathbb{Z})}$ has the property that any $d$ entries will together generate $S \widehat{L(n, \mathbb{Z})}$. This will follow from Theorem 12 and the fact (see page 442 of [5]) that whenever $n \geq 3$ and $d \geq 2$, the probability is positive that a randomly chosen $d$-tuple with entries from $S \widehat{L(n, \mathbb{Z})}$ will generate

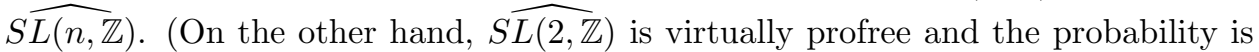
zero that a randomly chosen pair of elements will generate the group.)

Let $G$ be a profinite group that can be generated by $d$ elements. Let $\nu$ be the normalized Haar measure of $G$; abusing notation, we also denote by $\nu$ the corresponding measure on direct products of copies of $G$. For any $k \geq d$, let $\Omega(G, k, d)$ be the set of $k$-tuples of elements of $G$ with the property that every $d$ distinct entries together generate $G$. Let $P(G, k, d)=\nu(\Omega(G, k, d))$ and $P(G, d)=$ $P(G, d, d)$.

For each open normal subgroup $N$ of $G$, define $P(G, N, d)$ as follows. Let $\pi$ : $G^{d} \rightarrow(G / N)^{d}$ be the canonical quotient map. For any $x \in \Omega(G / N, d, d)$, let $P(G, N, d)$ be $\nu\left(\pi^{-1}(x) \cap \Omega(G, d, d)\right) / \nu\left(\pi^{-1}(x)\right)$. By Lemma 10 , this is independent of the choice of $x$, so $P(G, d)=P(G / N, d) P(G, N, d)$.

Lemma 10. Let $N$ be an open normal subgroup of $G$ and let $\pi: G^{d} \rightarrow(G / N)^{d}$ be the canonical quotient map. For any elements $x$ and $y$ of $\Omega(G / N, d, d)$,

$$
\nu\left(\pi^{-1}(x) \cap \Omega(G, d, d)\right)=\nu\left(\pi^{-1}(y) \cap \Omega(G, d, d)\right) .
$$

Proof. Once this is proven for all finite groups $G$, the result for profinite $G$ will pass through the inverse limit.

For finite $G$, we proceed by induction on the cardinality of $N$. Let $\mathcal{C}$ be the collection of proper subgroups $H$ of $G$ that satisfy $H N=G$. By induction, for each $H \in \mathcal{C},|H \cap N|^{d} P(H, H \cap N, d)$ equals the number of elements of $\pi^{-1}(x)$ with the property that every $d$ distinct entries together generate $H$. Thus,

$$
\frac{\nu\left(\pi^{-1}(x) \cap \Omega(G, d, d)\right)}{\nu\left(\pi^{-1}(x)\right)}=1-\sum_{H \in \mathcal{C}}\left(\frac{|H \cap N|^{d}}{|N|^{d}}\right) P(H, H \cap N, d),
$$

and the latter value is independent of the choice of $x$.

The following technical lemma will make short work of the main theorem:

Lemma 11. If $N$ is an open normal subgroup of $G$ then

$$
P(G, k, d) \geq P(G / N, k, d)\left(1-(1-P(G, N, d))\left(\begin{array}{l}
k \\
d
\end{array}\right)\right) .
$$


Proof. Clearly, if $\left(g_{1}, \ldots, g_{k}\right) \in \Omega(G, k, d)$, then $\left(g_{1} N, \ldots, g_{k} N\right) \in \Omega(G / N, k, d)$. So, assume $\left(g_{1} N, \ldots, g_{k} N\right) \in \Omega(G / N, k, d)$ and let

$$
\Lambda=\left\{\left(n_{1}, \ldots, n_{k}\right) \in N^{k} \mid\left(g_{1} n_{1}, \ldots, g_{k} n_{k}\right) \notin \Omega(G, k, d)\right\} .
$$

To prove the lemma it suffices to show that $\nu(\Lambda) / \nu\left(N^{k}\right) \leq(1-P(G, N, d))\left(\begin{array}{l}k \\ d\end{array}\right)$.

For each subset $I=\left\{i_{1}, \ldots, i_{d}\right\}$ of $\{1, \ldots, k\}$ with cardinality $d$, let $\Lambda_{I}$ equal

$$
\left\{\left(n_{1}, \ldots, n_{k}\right) \in N^{k} \mid\left\langle g_{i_{1}} n_{i_{1}}, \ldots, g_{i_{d}} n_{i_{d}}\right\rangle \neq G\right\} .
$$

The lemma then follows from the fact that $\nu\left(\Lambda_{I}\right) / \nu\left(N^{k}\right)=1-P(G, N, d)$ and $\Lambda=\bigcup_{I} \Lambda_{I}$.

Theorem 12. For a profinite group $G$ and a positive integer $d$, the following two conditions are equivalent.

(1) $P(G, d)>0$.

(2) $P\left(G, \mu_{d}(G), d\right)>0$.

The condition that $P(G, d)>0$ for some positive integer $d$ is equivalent to $G$ having polynomial maximal subgroup growth. This is a theorem of Mann [5] and Mann and Shalev [6].

Proof. Projection from $\Omega\left(G, \mu_{d}(G), d\right)$ to $\Omega(G, d, d)$ yields the implication of (1) from (2). We only show that (1) implies (2).

We want to prove that if $P(G, d)>0$ and $\Omega(G, k, d) \neq \emptyset$ then $P(G, k, d)>0$.

Because $G$ can be topologically generated by a finite number of elements, it possesses a countable descending chain of open normal subgroups, $N_{i}$, that has trivial intersection. Since $\lim _{i \rightarrow \infty} P\left(G / N_{i}, d\right)=P(G, d)>0$ and, for all $i, P(G, d)=$ $P\left(G / N_{i}, d\right) P\left(G, N_{i}, d\right)$, we see that $\lim _{i \rightarrow \infty} P\left(G, N_{i}, d\right)=1$. Therefore there exists a natural number $i$ such that $\left(1-P\left(G, N_{i}, d\right)\right)\left(\begin{array}{l}k \\ d\end{array}\right)<1$. Setting $N$ equal to $N_{i}$ in Lemma 11, we conclude that $P(G, k, d)>0$.

\section{REFERENCES}

[1] Bass, H.; Lazard, M.; Serre J.-P. Sous-groupes d'indice fini dans $S L(n, \mathbb{Z})$. Bull. Amer. Math. Soc. 70 (1964), 385-392.

[2] Blackburn, S. Sets of permutations that generate the symmetric group pairwise. J. Combin. Theory Ser. A 113 (2006), no. 7, 1572-1581.

[3] Britnell, J. R.; Evseev, A; Guralnick, R. M.; Holmes, P. E.; Maróti, A. Sets of elements that pairwise generate a linear group. J. Combin. Theory Ser. A. 115 (2008), no. 3, 442-465.

[4] Holmes, P. E.; Maróti, A. Pairwise generation of sporadic simple groups. Submitted for publication.

[5] Mann, A. Positively finitely generated groups. Forum Math. 8 (1996), 429-459.

[6] Mann, A.; Shalev, A. Simple groups, maximal subgroups and probabilistic aspects of profinite groups. Israel J. Math. 96 (1996), 449-468.

[7] Mennicke, J. L. Finite factor groups of the unimodular group. Ann. of Math. (2) 81 (1965), $31-37$.

[8] Neumann, B. H. Groups covered by permutable subsets. J. London Math. Soc. 29, (1954), $236-248$.

[9] Weigel, T. On the profinite completion of arithmetic groups of split type. Lois d'algèbres et variétés algébriques (Editor: M. Goze), Collection Travaux en cours. 50, (1996), 79-101.

Andrea Lucchini, Dipartimento di Matematica Pura ed Applicata, Via Trieste 63, 35121 Padova, Italy.

E-mail address: lucchini@math.unipd.it

Attila Maróti. Current address: The Mathematical Sciences Research Institute, 17 Gauss Way, Berkeley, CA 94720-5070, USA. Previous address: Department 
of Mathematics, University of Southern California, Los Angeles, CA 90089-2532, USA.

E-mail addresses: attilam@msri.organd maroti@usc.edu

Darren Semmen, Department of Mathematics, University of Southern California, Los Angeles, CA 90089-2532, USA.

E-mail address: semmen@usc.edu 